\title{
Petits planteurs et construction territoriale en Afrique noire : quels enjeux pour les cultures pérennes?
}

Oléagineux, Corps Gras, Lipides. Volume 8, Numéro 6, 587-92, Novembre - Décembre 2001, Dossier : L'avenir des cultures pérennes

Auteur(s) : Bernard CHARLERY DE LA MASSELIERE, Département de géographie, Université de Toulouse-Le Mirail, 31058 Toulouse Cedex 1.

Résumé : Les termes de référence de cette conférence ont rappelé l'importance majeure des cultures pérennes pour de nombreux pays en développement. Cette importance n'est pas seulement économique. Elle prend son sens au sein de l'évolution d'un système global où les exigences écologiques de la plante ainsi que les aptitudes bio-climatiques et pédologiques des milieux tropicaux interfèrent avec les conditions historiques du marché mondial, de la formation de sociétés paysannes ouvertes à l'innovation et de la destruction/reconstruction de l'État. Le mouvement d'introduction et de développement des cultures pérennes peut être considéré comme une véritable innovation, voire un fait de civilisation, inscrit dans une longue durée et dont on peut repérer les moments et lieux de l'intégration. Histoire souvent décrite, selon les pays, comme plus ou moins exemplaire, ayant apporté des transformations irréversibles de la société, jusqu'à produire des modèles spécifiques, comme au Kenya le modèle du " petit producteur capitaliste » de la Province centrale, des identités individuelles, à travers le statut de "planteur " comme en Côte d'Ivoire, ou des identités collectives, à travers la proclamation ethnique, comme en pays Bamileke. Réfléchir aujourd'hui sur l'avenir des cultures pérennes dans un contexte de crise nous place bien au cœur des temporalités, complexes, qui animent les éléments du système. François Ruf a bien montré pour le cacao comment la conjonction de facteurs fait passer une région de production, souvent en un très court laps de temps, de l'âge d'or à la crise, comment le transfert spatial des foyers de production vers de nouvelles régions, par une sorte d'accumulation extensive à l'échelle mondiale, a d'une certaine façon occulté, à l'échelle locale ou régionale, les principes élémentaires qui déterminent le cycle de l'innovation. Faut-il rappeler que les cultures pérennes sont des cultures d'exportation, rarement consommées sur le marché national, et que c'est donc de façon indirecte qu'elles participent aux impératifs d'une économie agricole à priorité vivrière. Cela pour dire que l'intégration de ces cultures dans les systèmes de production prend toute sa dimension, son sens - en termes d'innovation - non pas dans leurs procédures techniques ou commerciales propres, particulières, sectorielles, qui déterminent le fonctionnement de la filière, mais dans le rôle qu'elles jouent ou qu'elles ont joué dans le changement social au sens large. Cela pour dire avec plus de précision que l'avenir à long terme des cultures pérennes ne dépend pas d'abord d'un " raffinement " qui serait apporté aux conditions techniques et commerciales du secteur - en termes d'amélioration des rendements, de la compétitivité de la filière, etc. - mais bien de la façon par laquelle elles peuvent ou non accompagner ou relancer un processus plus large de recomposition sociale et territoriale rendu nécessaire par l'épuisement du modèle de développement. En ce sens, on peut dire que l'adoption des cultures pérennes n'a été qu'un préalable, un prélude ou un prétexte à des changements sociaux et territoriaux qui constituent la véritable innovation. La question peut être posée de façon plus brutale, pour l'Afrique du moins. Le développement des cultures pérennes a-t-il acquis une certaine autonomie par rapport à l'évolution des rapports de force au sein des sociétés, ou reste-t-il intrinsèquement subordonné à un moment particulier de leur histoire, rendu aujourd'hui caduc par l'étirement d'une crise paysanne durable ? Cette dernière proposition montre la difficulté 
dans laquelle nous nous trouvons dans la mesure où elle signifierait que, quelles que soient les améliorations apportées au fonctionnement du marché, la " durabilité " que celles-ci apporteraient aux cultures pérennes ne serait que temporaire et résiduelle, par rapport à des dynamismes qui trouveraient leur ressort ailleurs, dans d'autres types de production. Pour explorer cette question, il paraît utile de revenir sur les conditions du succès des cultures pérennes, mais aussi sur les limites de sa propre logique, et de les replacer dans un certain nombre de transformations qui apparaissent aujourd'hui comme déterminantes. On fera l'hypothèse qu'un nouvel ordre social et territorial est en train de se forger dans le cadre contraignant d'une dérive économique et financière - sinon sociale qui exacerbe la compétition pour l'accès aux ressources.

Summary : In sub-Saharan Africa, perennial crops have been introduced by the colonial governments. Their expansion led to dramatic changes in the organisation of land and areas, the structure of production relations, and the social-political construction. The perennial crops were at the root of a global, social, political economical, and geographical system, which had it own internal contradictions. Since the 80s, this system has been facing a severe crisis which poses many obstacles to its reproduction as investment for replanting and renewing plantations is required. Discussing the outlook for the future of the perennial crops means to redefine the whole condition which could allow smallholders not only to survive but to get a new status and new ways for social and economic promotion too. Market and crop chain liberalization, political and ethnic struggles, land shortage, urban employment crisis, etc., are the main constraints for securing a better and sustainable future for the producers.

Keywords : perennial crops, smallholders.

\section{ARTICLE}

\section{Le territoire, une construction sociale}

Pourquoi traiter la question des cultures pérennes à travers la notion de territoire ? II paraît nécessaire de préciser la nature et la raison d'un tel filtre qui, comme on peut le pressentir, met en jeu le rapport qu'un individu ou une société entretient avec son espace de vie et, par là, les rapports que les individus ou les groupes entretiennent entre eux.

On peut justifier cette approche à partir de l'hypothèse suivante : les cultures pérennes ont accompagné un processus de territorialisation, moment d'une histoire des sociétés locales et des États, et composante géographique d'un certain modèle de développement.

Comment peut-on comprendre ce processus de territorialisation?

* Les cultures pérennes marquent le paysage de leur empreinte. Elles sont le produit, à un moment donné, d'un certain rapport à la nature et d'un mode particulier de gestion des ressources. Elles produisent un écosystème particulier, à la combinaison d'éléments naturels (sols, pluviométrie, température, exposition, etc.) et d'éléments culturels et techniques (savoir-faire) entretenus, transformés et valorisés comme ressources dans le cadre des systèmes de production. Cette confrontation entre société et milieu prend corps dans le paysage qui est le reflet de la façon dont le producteur organise l'espace, fixe les territoires, domestique les flux, attribue fonction et vocation aux 
lieux. Ce sont des processus éminemment complexes par lesquels se joue non pas la "durabilité » d'un équilibre inerte mais bien toujours le développement de la société tout entière.

* Les cultures pérennes sont le vecteur matériel des dynamiques et des identités socio-culturelles. On trouve le lien entre identité et territoire dans les formes d'appropriation de l'espace : on connaît le rôle qu'ont joué les cultures pérennes dans l'accès à la terre, et les inégalités ou la différence entre les statuts qui en découlent. L'appropriation de l'espace passe aussi par le discours que la société tient sur elle-même, à travers des références symboliques qui orientent son mode de développement : en ce sens, la valorisation sociale et symbolique du statut du planteur renforce ce lien entre identité et territoire.

* Les cultures pérennes servent de support à la légitimation de hiérarchies institutionalisées ou de formes instituées de division du travail, à travers l'organisation de la filière, de la production à la commercialisation. En ce sens, les cultures pérennes ont participé directement à la construction d'un territoire national intégrateur des espaces locaux et ouvert sur l'international. Cette construction marque un certain degré d'institutionalisation du pouvoir qui n'est pas partout identique. Le rapport à l'État s'impose comme une constante historique, mais aussi comme une des conditions de définition des sociétés paysannes elles-mêmes. Ces types de relations définissent un système qui est d'abord national par le fait qu'il se déploie sur un territoire que, localement, les producteurs occupent et transforment, mais dont l'État gère les affectations - avec l'aide de l'entreprise privée - et qu'il encadre en amont comme en aval. L'État ayant en charge l'allocation territoriale des ressources et des types de cultures, c'est en fonction de sa puissance, de son identification plus ou moins forte à une classe de producteurs et de sa relative dépendance vis-à-vis des revenus tirés des cultures pérennes que, localement, s'engagent les initiatives des producteurs.

* Les cultures pérennes sont à l'origine de la création de réseaux ou sont le prétexte de leur activation : réseaux marchands, mais aussi réseaux sociaux, de parenté, de classe, de clientèle, réseaux d'information et de communication. Ces réseaux sont plus ou moins transparents ; ils créent souvent des sortes d'opacité dans le fonctionnement rationnel des filières, qui favorisent le jeu de la recomposition des pouvoirs. Ils tissent une trame qui peut être ou non en concordance avec l'organisation des territoires ou avec l'organisation des grands appareils qui règlent le fonctionnement de la filière. Réseaux et territoires se présentent donc comme un ensemble où circule le pouvoir, un pouvoir qui n'est pas définitivement acquis mais qui est exercé, c'est-à-dire sans cesse négocié à travers des relations dissymétriques. Dans un contexte de crise des appareils, ils peuvent favoriser ou, au contraire, handicaper ou parasiter la nécessaire réorganisation des filières.

À partir de ces quelques propositions, que nous essaierons de préciser par la suite, nous pouvons déjà comprendre que l'avenir des cultures pérennes relève d'un double jeu relativement complexe : d'une part, des pratiques symboliques, identitaires, autour des différentes cultures qui fondent les formes d'appartenance au territoire et gèrent l'accès aux ressources et, d'autre part, des modes d'accumulation publique ou privée, des modes de développement local ou national, lesquels légitiment des stratégies individuelles et collectives dont l'assise doit être sans cesse assurée.

On voit donc comment les cultures pérennes ont favorisé la mutation des systèmes de production localisés et la constitution d'identités sociales, appelés à se redéfinir dans un contexte radicalement nouveau. Comment l'avenir des cultures pérennes dépend-il aujourd'hui de conditions qui débordent le cadre stricto sensu de l'organisation de la production ou de la commercialisation du produit. II faut replacer nos perspectives dans le cadre des transformations et de la valorisation de l'environnement 
local de la production, mais aussi des possibilités offertes ou non aux individus et aux groupes d'assurer leur promotion, de redéfinir leur rapport avec l'autre (I'homme par rapport à la femme, une génération par rapport à l'autre, un groupe ethnique par rapport à un autre, etc.) et dans le cadre de la réorganisation de l'État.

\section{Le petit producteur, fer de lance du contrôle territorial : paradoxes et limites d'un modèle}

À l'aube de la colonisation, l'Afrique a globalement été confrontée à une remise en cause des structures sociales et territoriales de groupes, communautés ou sociétés très diversifiés, ainsi que des rapports et des modes d'échanges qu'ils entretenaient. Ceux-ci traversaient une situation de crise et d'indétermination spatiale dans laquelle la colonisation s'est engouffrée. La colonisation a exercé un pouvoir d'unification en réorientant et en mobilisant les anciennes structures dans les perspectives de la nouvelle économie, celle de la production. Unifier les espaces, orienter les flux des personnes et des biens, investir les terres agricoles et les terres vierges des nouvelles productions, tels ont été les ingrédients de cet engrenage territorial à travers lequel il faut relire le développement des cultures pérennes. L'équilibre du nouvel édifice administratif et territorial s'est fondé sur l'engagement des paysans dans le nouvel effort agricole et sur leur capacité à modifier leur mode de production sur la longue durée. Cet engagement, nous le savons, a progressivement consacré, à côté des grandes plantations, l'exploitation familiale comme l'unité de production de référence. Il a aussi inséré les producteurs dans la société globale, en imposant toute une série de contraintes dans l'orientation des trajectoires individuelles et collectives.

À partir de 1930, s'est mis en place un véritable système, voire un modèle, qu'on peut qualifier de " colonial " ou modèle de développement, qui va entrer en crise à partir des années 80 , à un moment où, par ailleurs, les plantations vieillissant, le marché devenant instable, un effort supplémentaire est demandé aux producteurs afin de sortir d'une logique purement rentière du produit d'initiatives anciennes.

En regard des questions qui sont posées dans les termes de référence de cette conférence, sur les dégradations écologiques, les migrations, la durabilité de la production, l'accès à la terre, la transmission patrimoniale, le marché, je voudrais montrer, rapidement, comment ces différents éléments, ayant produit leur propre blocage, ne sont plus aujourd'hui opératoires et pourquoi, alors qu'ils ont été facteurs du succès des cultures pérennes, il devient difficile, voire illusoire, de les réactiver dans une perspective de relance de la production.

\section{Conquête foncière, frontière sociale}

Il est indéniable que, avec les cultures pérennes, le capitalisme et l'économie mondiale ont fait irruption dans le secteur paysan d'autosubsistance et l'ont profondément transformé. Avec les cultures pérennes, le système paysan est entré dans une phase, un moment de sa mobilité, entraînant ses acteurs au-delà de leurs territoires de référence - à la recherche d'un revenu monétaire rendu indispensable par la coercition du colonisateur, l'exiguïté des terres ou encore la fragilité du secteur vivrier devant les aléas climatiques et la croissance démographique - ou de l'autre côté des frontières agricoles existantes, à la conquête de la terre qui est souvent aussi une conquête sociale. Les cultures pérennes en tant que marqueurs fonciers ont créé une véritable révolution dans les modes d'accès à la terre, et c'est là sans doute l'un des facteurs décisifs de leur succès.

En même temps et dans le même mouvement s'est opérée la capture de la paysannerie par l'État qui l'a utilisée comme fer de lance de sa propre prise de contrôle du territoire de même qu'il a utilisé les 
cultures de rente comme base de sa propre « économie sociale ». Ces différents mouvements ont suivi des trajectoires complexes et diversifiées : mouvements temporaires ou définitifs, intermittents ou continus, centrifuges ou centripètes, etc., mais tous passant par les cultures pérennes, y puisant une nouvelle dimension.

Les opportunités créées par la fin du monopole étranger ou par l'ouverture ou la libération de nouvelles terres ont accentué le mouvement en le consolidant, en le fixant et en assurant ses racines paysannes. La conquête de la terre par l'intégration des zones périphériques, le fractionnement des domaines fonciers ou le démantèlement des grandes exploitations a confirmé un mouvement de promotion, de transformation et de différenciation du secteur paysan, qui scelle son intégration à l'économie mondiale. Les cultures pérennes apparaissent comme des éléments fondateurs d'une société qui évolue et se différencie profondément et d'un espace qui se conquiert, se " produit " au sens large du terme.

Le développement des cultures pérennes a donc été assuré grâce à la disponibilité de réserves foncières et grâce à la souplesse des systèmes fonciers traditionnels dans l'intégration des migrants, voire en certains cas à une réelle indétermination - au sens étymologique - des formes d'appropriation. Ces différentes situations ont ouvert des frontières agricoles, autorisant de nouvelles affectations des espaces. Mais qui dit pérenne dit une certaine permanence. Comme l'a parfaitement décrit François Ruf à propos du cacao, qu'advient-il après la consommation initiale, souvent spontanée, des espaces ? Les cultures de rente permettent au colonisateur et à l'État de fixer leur propre territoire, de contrôler et d'orienter l'émigration traditionnelle. Ces orientations vont se traduire par une saturation foncière, générale dans les hautes terres d'Afrique de l'Est vers les années 60. Dans la question qui nous intéresse, il est utile d'insister sur ce blocage foncier, d'autant plus, nous le verrons, qu'il infléchit fortement la redéfinition des identités locales et régionales. À l'échelle des exploitations, le rapport foncier est de plus en plus réduit à la relation simple entre une unité sociale et une unité territoriale par la promotion de la petite propriété privée. L'espace local va se trouver inscrit dans une logique de la finitude qui bloque les transformations nécessaires des rapports sociaux et ôte toute souplesse à la nécessaire recomposition des unités de production.

Enfermés dans un maillage territorial et identitaire contraignant, les paysans doivent alors chercher ailleurs que dans l'élargissement de leur environnement productif les conditions de leur développement. Les petits producteurs, dans leur majorité, vont réagir, d'une part, en démultipliant les filières de mobilité spatiale et sociale (vers la ville) et, d'autre part, en intensifiant sans assurance de rentabilité leur investissement sur place ou en ouvrant des fronts intérieurs qui fragmentent l'espace en petites unités hétéroclites de moins en moins rentables et soumises aux aléas des contingences individuelles. On est loin ici des " bases nécessaires à la formulation d'une politique susceptible d'assurer un avenir meilleur et durable pour les producteurs $»^{1}$.

\section{Rapport à la nature et rationalité technique}

L'économie de marché a totalement bouleversé le rapport qu'entretenaient les sociétés agraires avec la nature. Pour faire vite, je rappellerai que les sociétés rurales africaines étaient, selon l'expression de George Balandier, « plus soucieuses de produire du sens que de la richesse matérielle ». Avec la colonisation, on assiste à un effacement de cette nature sociale et symbolique de l'espace, réduit à un simple objet matériel, à un simple outil de production. Ce fut le rôle de l'administration technique de réaliser cette transformation, d'où l'importance prise, dans la production du territoire, par les techniciens. L'idéal productiviste - dont les cultures pérennes sont une forme de matérialisation - a 
ainsi fonctionné comme un formidable modèle de référence dont les contradictions ont été appréhendées par l'agriculteur de plus en plus au seul niveau individuel et presque uniquement en termes de manques : manque de savoir-faire, manque de terres, manque de moyens, d'argent, d'intrants, de temps, etc. II s'agissait bien d'un projet de subordination, sinon de domination, dont l'objectif, avoué ou non, restait bien la mobilisation du travail paysan et l'engagement dans un nouveau procès brisant les hiérarchies et les solidarités traditionnelles.

La colonisation, en contraignant l'innovation sociale endogène dans une logique productiviste, a séparé la gestion du milieu de ses principes sociaux et symboliques régulateurs. L'espace est devenu une simple addition de lieux réduits à un usage productif vers lesquels a été orienté et détourné le mouvement social, lui-même ramené au simple déploiement de la force du travail. Si l'on veut aujourd'hui remobiliser les capacités d'innovation sociale des paysans, c'est une nouvelle représentation de l'espace, de la nature, qu'il faut produire, sachant que ce qui est en cause c'est le statut du travail de I'homme sur la matière, l'exploitation de la nature et l'exploitation du travail devant être intégrées dans un même processus. On voit cependant la difficulté qu'il y a aujourd'hui à inscrire l'avenir des cultures pérennes dans un cadre qui lui serait historiquement étranger.

\section{Migrations et fixations identitaires}

Intensification agricole et mobilité ont été très souvent associées : l'exemple le plus significatif est sans doute trouvé au Kenya où les premiers agriculteurs engagés dans la culture du caféier ou du théier ont acquis les moyens d'une accumulation grâce au travail salarié dans les administrations, les missions religieuses ou les grandes plantations. S'est ainsi instaurée une économie du travail migrant, salarié, temporaire et saisonnier, soit vers le secteur rural, soit vers les centres urbains, religieux ou administratifs : I'articulation souple entre les différents espaces, secteur d'autosubsistance et secteur moderne, permettait de conserver un coût relativement bas pour la main-d'œuvre et un ajustement permanent de l'emploi aux fluctuations des marchés. C'est la diffusion de la monnaie qui a sans aucun doute renforcé la nécessité pour les agriculteurs de recourir au travail migrant. II faut souligner la capacité paysanne à transformer ce capital en statut social sur la base de l'appropriation foncière : on peut trouver de nombreux exemples où les plantations ont permis aux détenteurs des droits fonciers mais aussi aux clients fonciers de consolider leur propre assise sociale et où l'argent du café a permis d'investir dans les secteurs plus valorisants de la fonction publique ou du commerce et dans la scolarisation des enfants.

Les transformations qu'engendre la nouvelle mobilité sont d'abord sociales. Elles se traduisent surtout par un affaiblissement des groupes locaux qui, entre autres, renforce les contraintes dans l'emploi de la force de travail : migrations des jeunes, migrations des clients fonciers, migrations des femmes. La remise en cause des pouvoirs traditionnels, comme la perte d'une autorité collective sur les attributions foncières par la dévalorisation symbolique du rapport à la terre, renforce par ailleurs les tendances particularistes.

On touche là un des points sans doute les plus importants de l'évolution du rapport au territoire. La logique migratoire, dont le développement des cultures pérennes est l'un des principaux ressorts, a été un facteur de la construction de l'espace national et donc de l'État. Avec le développement des cultures pérennes et la mobilité qui lui est associée, on peut dire que la dynamique paysanne a investi un espace plus vaste que l'espace local mais qui lui est encore étranger, à savoir celui d'une " citoyenneté " qui reste insaisissable et ambiguë. Le succès des cultures pérennes d'exportation peut laisser supposer que s'est trouvée une sorte de " compromis territorial », rendu possible à un moment 
donné par l'adéquation entre les nécessités économiques et politico-spatiales de l'État, les besoins en main-d'œuvre et les nécessités de développement et de promotion des sociétés rurales et leurs élites. On a pu même écrire, à propos du Kenya, que ce succès a été la garantie d'un véritable contrat social. Ce compromis a engagé un processus d'identification nationale inachevé. En effet, étant donné I'histoire particulière de la création des États en Afrique noire, l'idéologie nationale contient des concessions importantes aux particularismes locaux, ethniques, claniques, religieux ou régionaux. L'État a contracté envers ces différents groupes une dette qu'il honore en redistribuant une part de la rente, soit de façon plus ou moins équilibrée quand le gouvernement est le produit d'un consensus interethnique ou interrégional, soit de façon déséquilibrée - source de conflits - quand il est l'émanation d'un groupe social ou d'une région particulière. L'investissement territorial de l'État est toujours la résultante d'un rapport de forces, éminemment variable.

La diminution globale de la rente tirée des cultures pérennes et, donc, de la capacité de redistribution de l'État, ajoutée dans de nombreux cas au blocage foncier et à la crise de l'emploi salarié, a aujourd'hui renforcé la compétition entre les groupes et les individus, exacerbé les particularismes sociaux et favorisé le développement des inégalités.

Dans ce contexte de baisse, voire d'incertitude généralisée de la rente tirée des cultures pérennes, tous les ressorts du développement social des producteurs sont cassés. Les conflits fonciers sont l'expression d'une crispation identitaire, où le rapport à la terre ne porte plus ce développement social, n'offre plus la flexibilité nécessaire à une recomposition des territoires, mais témoigne de logiques d'exclusion et de pratiques conservatoires sans autre finalité que l'affirmation ethnique ou nationale en soi.

\section{Patrimoine : le transfert d'échelle}

Ce que nous venons de dire remet également en cause toute la logique patrimoniale du rapport entre les générations. Les cultures pérennes ont redessiné le cadre patrimonial dans lequel l'économie domestique se déploie de deux façons : en faisant de la parcelle de caféier, de théier, etc. :

- le moyen d'élargir l'univers social des " pères " par leur inscription dans un réseau de clientèle à travers l'organisation de la filière. L'articulation des dynamiques individuelles au projet national est passée essentiellement par l'encadrement des filières et l'organisation des échelons administratifs. Le système coopératif en particulier a intégré tous les éléments de la fonction patrimoniale assurée autrefois dans le cadre clanique ou lignager. Il est devenu le lieu privilégié de l'alliance, de la solidarité, de la redistribution, de la protection et de la promotion. Les liens tissés ici ou là entre le mouvement coopératif et les mouvements nationalistes témoignent, à l'aube des indépendances, de cette articulation entre les échelles et les niveaux d'encadrement ;

- le témoin de la transmission intergénérationnelle, en particulier sur les terres nouvelles gagnées aux marges des terroirs vivriers ou sur de nouveaux fronts. Mais très tôt, ce processus de transmission a changé d'échelle, quittant les limites de l'exploitation familiale. On sait combien les revenus des cultures pérennes ont servi à financer la scolarisation des jeunes, leur ouvrant des voies de sortie de l'agriculture, en particulier dans les métiers urbains, de la fonction publique entre autres. Cette priorité de la ré-allocation des revenus - qui, rappelons-le, sont restés, pour la plupart des petits producteurs, relativement modestes - s'est faite au détriment de l'investissement direct dans les cultures pérennes et de l'anticipation de leur renouvellement au bout du cycle végétatif des arbustes. 
Aujourd'hui, le matériel végétal a vieilli et, dans de nombreux endroits, il n'est plus productif. Par ailleurs, on sait à quel point l'intégration de la culture à un cadre national a entraîné un détournement de la rente vers des réseaux non directement productifs. La logique patrimoniale est devenue un instrument d'appropriation privative du bien public au profit des factions au pouvoir, un instrument également d'accès à un capital " anonyme ", c'est-à-dire dissocié des systèmes de production qui ont permis l'accumulation primitive.

En de nombreux pays, la débâcle des grandes coopératives et la réduction drastique de leur capital interdisent toute relance de la production. La baisse des ressources de l'État sape la légitimité de son intervention et remet en cause les mécanismes qui assuraient l'insertion, inégale mais socialement constructive, des individus dans le maillage du tissu social et politique. Les crispations autour de l'appartenance ethnique ou nationale détournent le processus d'identification - qui a été un des ressorts du succès des cultures de rente - vers un champ juridico-politique autonome et fonctionnant en vase clos. Enfin, la crise de l'emploi public et la paupérisation urbaine désagrègent les liens de solidarité et les petits réseaux entre villes et campagnes : cette individualisation des urbains par rapport à leur terroir d'origine rompt la chaîne patrimoniale qui avait justifié l'investissement dans l'économie de plantation. En bref, il n'y a plus grand chose à transmettre sur la base même du système des cultures pérennes.

\section{La question des femmes : l'espace vivrier}

On rappellera rapidement ce qui est bien connu et ce qui a été bien analysé sur l'évolution des rapports entre les hommes et les femmes à partir de la diffusion des cultures pérennes. On sait combien celles-ci ont permis d'asseoir l'identité masculine au sein de l'exploitation. On sait également que le succès des cultures pérennes a été rendu possible grâce à une surexploitation du travail féminin. Les cultures pérennes ont modifié l'insertion des hommes dans le champ social global. Elles ont reproduit, en la renouvelant, l'individuation des hommes par l'acquisition d'une indépendance monétaire qui a modifié la nature du contrat domestique. Elles ont également créé les conditions d'une compétition pour l'accès aux ressources dans le cadre de leur rapport aux cultures vivrières, domaine des femmes. Les cultures pérennes ont transformé les équilibres antérieurs en réduisant la marge de manœuvre des femmes, de plus en plus cloisonnées dans les limites exiguës de l'exploitation, par l'alourdissement de leurs tâches traditionnelles et par l'ajout de tâches nouvelles dans la parcelle de culture pérenne.

Cependant, on peut aussi préciser que, par leur travail voire leur surtravail, les femmes ont en général su conserver des formes d'indépendance par un investissement renforcé dans les cultures vivrières ou l'élevage. Ce surtravail a par ailleurs renforcé l'identification de leur statut au territoire, alors que les hommes, par les cultures pérennes, ont orienté leurs propres stratégies vers de nouveaux réseaux d'échange qui, eux, sont extérieurs au territoire. On peut voir là les prémices d'un découplage entre le champ des femmes et le champ des hommes. La nouvelle attention portée aux femmes dans les projets de développement agricole n'est pas étrangère à cette situation.

La baisse de la rente tirée des cultures pérennes et la désorganisation des filières, d'une part, et l'ouverture de nouveaux marchés vivriers urbains et l'activation de réseaux commerciaux souvent traditionnels, d'autre part, placent les femmes aux avant-postes d'une redynamisation de la production agricole et de la réinsertion des territoires productifs à l'espace national, voire régional. Les femmes ayant été exclues, sauf situations particulières, des cultures pérennes, on voit 
progressivement les cultures vivrières ou maraîchères gagner les parcelles de cultures pérennes. La disparition du café arabica sur les hautes terres de l'Ouest du Cameroun, qui alimente en produits frais les marchés régionaux au-delà des frontières mêmes du pays, est un des exemples les plus spectaculaires de cette mutation qui n'est pas seulement économique mais aussi sociale.

Quelle leçon peut-on tirer de cette longue analyse qui, je le souligne, s'appuie sur des situations particulières n'ayant pas nécessairement force de loi générale mais dont l'addition permet au moins d'éclairer nos perspectives, sur l'Afrique en particulier ? Les cultures pérennes ont construit un véritable système, social et territorial, dans le fonctionnement duquel elles ont puisé les ressorts de leur développement. Réfléchir sur l'avenir des cultures pérennes, c'est donc en repenser toutes les composantes complexes : d'où la difficulté de l'exercice auquel nous nous livrons ici. II me paraît clair, pour me répéter, que toute solution partielle, technique ou commerciale, de relance des cultures pérennes ne prendrait pas la mesure du problème. Mon propos n'est pas très optimiste. Peut-on tracer aujourd'hui les voies de ce qui devrait être une révolution sociale, organisationnelle et territoriale où les cultures pérennes pourraient retrouver un rôle plein et entier d'innovation ?

\section{La nouvelle donne territoriale}

Comment peut-on aujourd'hui replacer l'avenir des cultures pérennes dans le cadre du nouveau jeu territorial imposé par le libéralisme contemporain ? En m'appuyant sur un excellent article de Daniel Hiernaux-Nicolas ${ }^{2}$, je rappellerai brièvement quels en sont les fondements avant de proposer quelques pistes sur les conditions actuelles de l'innovation sociale à laquelle pourrait participer une relance des cultures pérennes.

La globalisation en œuvre depuis deux décennies a créé les conditions d'une transformation radicale des structures essentielles sur lesquelles s'appuyait le mode antérieur de développement, où l'espace apparaît comme un aspect essentiel de la configuration du modèle économique et social proposé par le libéralisme contemporain. Même si, en matière territoriale, prévaut encore un discours planificateur, égalitariste et développementaliste, la réalité va à l'encontre d'un tel projet.

Le développement en général, et celui des cultures pérennes en particulier, s'est construit sur la base d'une conception spatio-temporelle linéaire. L'espace a été soumis à une logique faite de continuité et d'expansion. L'aspiration à la continuité a encouragé les États modernes à l'intégration territoriale, qui devait être obtenue grâce aux actions colonisatrices et à de grandes articulations territoriales, en particulier grâce à l'intervention de l'État-nation, créateur de l'espace-nation. Nous avons vu à quel point les cultures pérennes ont servi cet objectif, à travers leur expansion dans le temps et dans l'espace, et l'usage de la capacité du petit producteur à occuper cet espace, à l'investir des modes de fonctionnement et des codes du développement. Nous avons vu que l'État n'avait plus les moyens de remplir ce rôle ; dans les pays en développement, ces moyens lui ont été retirés dans le cadre des PAS, et sa légitimité même a été contestée au nom d'une certaine efficacité, voire d'une certaine morale de gouvernance.

Mais, il faut aller au-delà de cette simple critique de l'État. La globalisation bouleverse le rapport établi entre les territoires dans la mesure où le niveau d'intégration et de concurrence s'établit aujourd'hui prioritairement à l'échelle mondiale. Comme le développement capitaliste s'était appuyé sur la flexibilité et la mobilité du travail, il suppose également une certaine flexibilité des territoires. Cette flexibilité est rendue nécessaire dans la mesure où toute activité nécessite un ancrage territorial plus ou moins prononcé, plus prononcé pour les activités agricoles. L'accumulation et le profit à l'échelon mondial se fondent sur la séparation des espaces en fonction de leur niveau de compétitivité 
territoriale qui correspond à leur capacité à jouer un rôle spécifique dans le système mondial. Comme le travail a dû être " libéré " de toute entrave à sa mobilité, la flexibilisation territoriale repose sur l'élimination progressive des mécanismes de contrôle et d'organisation du territoire. Les politiques de décentralisation et de libéralisation des filières ont ainsi appelé une revalorisation des forces locales aptes à engager les territoires régionaux, libérés du poids du contrôle étatique (qui a un coût), dans cette nouvelle configuration. La fragmentation consiste donc à conférer à chaque unité territoriale un poids spécifique non subordonné à celui des autres, c'est-à-dire un rôle et un éventail d'activités définis et contrôlés en fonction de l'économie à l'échelle mondiale. Les territoires des cultures pérennes, qui sont d'abord des cultures d'exportation, ont bien un rapport avec ce niveau d'intégration mondiale. L'enjeu se situe bien ici entre exclusion ou intégration.

Cette fragmentation des territoires suppose parallèlement la reconstitution de réseaux qui, à la fois, favorisent le développement des activités à l'échelle mondiale et garantissent le fonctionnement des différentes parties, ce qui suppose un effort de coordination et d'intégration à une échelle souvent supranationale. On se situe donc à la croisée, d'une part, de la revalorisation ou de la revitalisation des caractéristiques fondamentales d'espaces spécifiques et, d'autre part, de la création de réseaux (d'information, de circulation des techniques, de commercialisation, etc.) qui transgressent les limites de ces espaces spécifiques.

La revalorisation de l'échelle locale met en jeu des facteurs non économiques dans la mesure où elle est le lieu où se définissent des relations économiques, sociales, culturelles et politiques qui ne s'inscrivent pas dans la sphère du marché. Nous en avons donné quelques exemples. L'échelle locale a donc une certaine autonomie par rapport au marché, mais les modes de relations qui y sont établis déterminent sa capacité ou non à participer au nouveau développement. Tel est donc le cadre général dans lequel il faut penser l'avenir des cultures pérennes, si avenir il y a. La difficulté vient du fait que les situations locales sont « improbables » et ne relèvent pas d'un modèle unique.

On voit donc que la question est bien celle de la reconstruction de réseaux locaux qui, pour être efficients, devraient permettre aux producteurs de s'inscrire dans de nouvelles trajectoires de promotion sociale. Cela demande d'importants investissements non directement productifs à court terme, permettant entre autres une meilleure professionnalisation des fermiers et une prise en compte de leur environnement global. L'État s'est déjà désengagé d'un tel objectif. Le secteur coopératif n'a pas les ressources financières pour assurer ce rôle traditionnel. Les opérateurs privés restent dépendants de leurs objectifs de rentabilité imposés par le marché et qui n'intègrent pas ce type d'investissement.

Les vestiges du territoire des cultures pérennes portent les stigmates d'une conception coloniale du lien entre produit, filière et territoire avec laquelle il s'agit de rompre. Les vertiges du marché libéral n'offrent pas la stabilité nécessaire à des politiques menées sur le long terme et désorientent, plus qu'ils n'assurent, la démarche des producteurs. Les nouvelles formes de la concurrence territoriale sont pourtant l'occasion de redéfinir l'architecture des relations qui déterminent l'originalité et la compétitivité d'un territoire riche en ressources naturelles et humaines. Elles appellent une revalorisation des individus et de la communauté qui restent garants de la fertilité des terroirs de production. Elles engagent les forces transnationales à redéfinir, pour leur propre réussite, leur inscription au lieu.

* Ce texte traite des cultures pérennes en général, mais il s'appuie sur une expérience et des études de terrain menées plus particulièrement chez les petits producteurs de café des hautes terres d'Afrique. 
Notes :

${ }^{1}$ Texte introductif de la plaquette « dernière annonce du colloque de Yomoussoukro ».

${ }^{2}$ Daniel Hiernaux-Nicolas. Fondements territoriaux du libéralisme contemporain. Revue Tiers Monde $1999 ; 157: 107-20$.

\section{REFERENCES}

BART F, CHARLERY B, CALAS B (1998). Caféicultures d'Afrique orientale, territoires, enjeux et politiques. Paris, Nairobi : Karthala-IFRA ; $368 \mathrm{p}$.

CHARLERY de la MASSELIÈRE B, éd. (1997). Petits producteurs et filières agricoles au Kenya. IFRA Les Cahiers, 7-8: $138 \mathrm{p}$.

CHARLERY de la MASSELIÈRE B (1999). Territorialités multiples et conflictuelles : réponses paysannes à la crise des campagnes africaines. In : BONNEMAISON J, CAMBREZY M, QUINTY-BOURGEOIS L, éds. Les territoires de l'identité. Le territoire lien ou frontière ? Paris : L'Harmattan (coll. Géographie et culture) : 245-58.

CHARLERY de la MASSELIÈRE B (2000). À propos de la question foncière sur les Hautes Terres de I'Afrique orientale. In : NICOLAÏ H, PÉLISSIER P, RAISON JP, éds. Un géographe dans son siècle. Actualité de Pierre Gourou. Paris : Karthala/Géotropiques : 199-203.

CHARLERY de la MASSELIÈRE B (2001). Perte de la "frontière " et dérivations identitaires : les paysanneries des hautes terres d'Afrique de l'Est, entre confinement local et transgression des territoires. In : BART F, MORIN S, SALOMON JN, éds. Les montagnes tropicales. Identités, mutations, développement. Bordeaux : Dymset/Cret : 561-78.

RUF F (1995). Booms et crises du cacao. Les vertiges de l'or brun. Paris : ministère de la Coopération, Cirad-SAR, Karthala ; $459 \mathrm{p}$.

TULET JC, CHARLERY B, BART F, PILLEBOUE J (1994). Paysanneries du café des hautes terres tropicales. Paris : Karthala ; $310 \mathrm{p}$. 


\section{Illustrations}

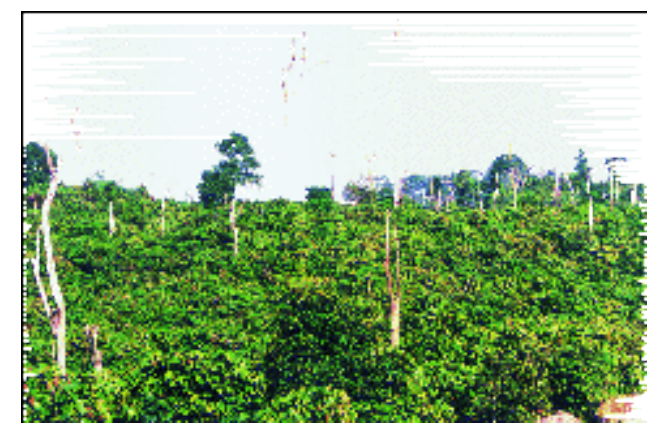

Photo. Plantation de cacao sur front pionnier (crédit photo : F. Ruf).

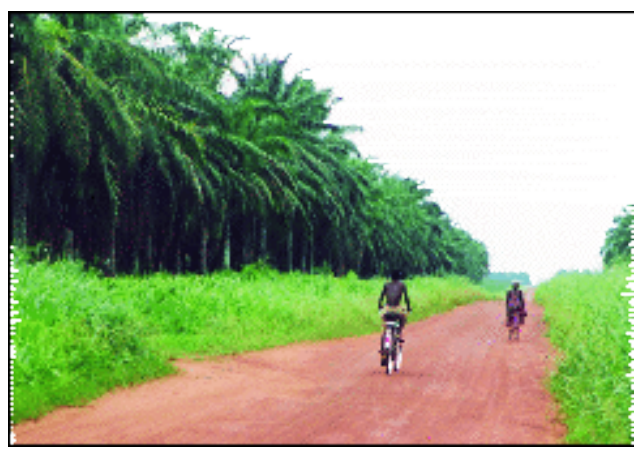

Photo. Plantation de palmiers sélectionnés (crédit photo : J. Muchnick). 


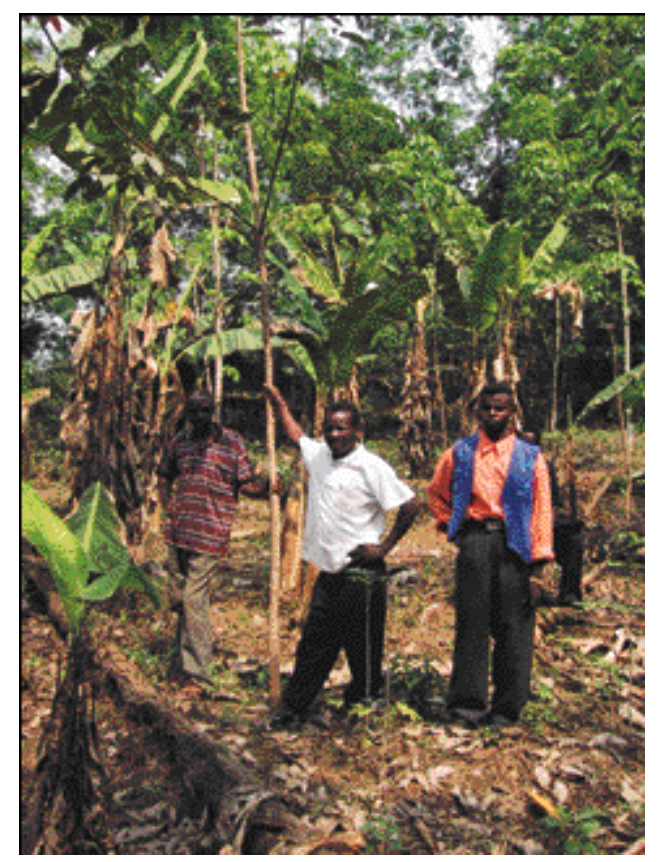

Photo. Hévéaculteurs (crédit photo : J. Sainte-Beuve). 